\title{
Optimization of Generalized S-curve Trajectories for Residual Vibration Suppression and Compliance with Kinematic Bounds
}

\author{
Luigi Biagiotti, Claudio Melchiorri
}

\begin{abstract}
This paper proposes a new optimization algorithm that assures the minimum possible duration of generalized S-curve trajectories compliant with kinematic limitations and capable of suppressing residual vibrations when tracked by a resonant plant. Thanks to the possibility of generating such kind of trajectories with a chain of filters, called smoothers, each one characterized by a single parameter, i.e. the duration $T_{i}$ of its impulse response, the optimization process aims at minimizing the order of the trajectory, and accordingly the number of smoothers in the chain, and leads to rest-to-rest trajectories that, under the given specifications, cannot be made shorter in time. Therefore, the structure of the trajectory is not predetermined but is the outcome of the proposed algorithm together with the optimal parameters defining it. The effectiveness of the proposed approach is proven by applying the designed trajectories to an experimental setup based on a flexible link.
\end{abstract}

Index Terms-S-curve trajectories, Minimum-time trajectories, Residual vibrations suppression, Smoothers, Shaping filters.

\section{INTRODUCTION}

The development of motion control systems able to plan fast and accurate trajectories for electromechanical systems, including automatic machines and robots, is a key factor for increasing productivity in many industrial fields. Such systems are designed to pursue different goals, often conflicting, like e.g. minimize the duration of the generated motions, assure the compliance with the kinematic limits imposed by the actuator, avoid exciting natural vibration modes of the mechanical structure. Besides these objectives, the additional requirement of online trajectory generation/modification is often needed.

The solutions are typically based on generalized S-curves, i.e. motion profiles composed by polynomial functions properly joined to assure that, besides velocity and acceleration, also the jerk and even higher derivatives are limited, see [1], [2] among many others. The main difference among the various trajectories available in the scientific literature or used in the industrial practice consists in their different design philosophies, that can be categorized into three main groups:

- trajectories defined via closed-form expressions [2], [3], [4];

- trajectories defined via numerical methods [5], [6], [7];

- trajectory obtained as the output of dynamic systems [8], [9], [10], [11].

L. Biagiotti is with the Department of Engineering "Enzo Ferrari", University of Modena and Reggio Emilia, via Pietro Vivarelli 10, 41125 Modena, Italy, e-mail: luigi.biagiotti@unimore.it.

C. Melchiorri is with the Department of Electrical, Electronic and Information Engineering "Guglielmo Marconi", University of Bologna, Viale Risorgimento 2, 40136 Bologna, Italy, e-mail: claudio.melchiorri@unibo.it.
Each of these different methods offers pros and cons in terms of computational burden, maximum order of the trajectory and consequently maximum number of constraints that can be handled, limitations (like e.g. the possibility to plan only restto-rest trajectories or to handle only symmetric constraints, etc.), online implementation. A quite comprehensive overview of the features of the different techniques can be found e.g. in [7]. From the scheme reported in that paper, it comes out that the optimality, i.e. the minimum duration, is guaranteed only in case of low-order (2nd and 3rd order) trajectories, while time-optimality remains an open problem in the general case. Moreover, most of the methods available in the literature deal with kinematic constraints, i.e. velocity, acceleration, and jerk bounds, while additional specifications, like for instance the suppression of the residual vibration at a specific frequency, are often neglected or not addressed explicitly. For instance, the jerk limitation/minimization, is generally used to avoid the excitation of resonance frequencies that may affect generic mechanical systems, ranging from robot manipulators [12], [13] to industrial machines [14]. An interesting attempt to mix the compliance with bounds on velocity and acceleration and an explicit frequency constraint aiming at suppressing a residual vibration can be found in [15], [16], where the tuning of the S-curve parameters in the Laplace domain is proposed, while the usual approach for vibration suppression is based on filtering techniques (input shapers [17], [18], [19] or FIR filters [20], [21] among others) of a reference trajectory already compliant with bounds on velocity, acceleration, etc. Obviously, this type of method does not allow a global optimization of the final reference signal with respect to its duration. A general framework for the design of an $\mathrm{S}$ curve trajectory generator able to deal with both kinematic and frequency constraints have been proposed in [22]. This novel framework is based on the iterated application of basic elements, called smoothers, that are finite impulse response filters but (initially) defined in the continuous-time domain. The proposed technique allows dealing with different types of constraints from a unitary perspective and, in principle, allows to considerably reduce the duration of the final trajectory. However, this optimization problem has been not completely solved yet. In [23], an algorithm that minimizes the duration of the trajectory under kinematic constraints is given, but the integration with the frequency constraints does not lead to the global optimum since the degrees of freedom, that characterize the problem, have been not fully exploited (this point is clarified in Sec. IV]. This is instead the main novel 
contribution of the present paper, together with some other minor results. More into detail, the novel contributions of this work are to:

- clarify the origin of the constraints that must exist among the parameters of a chain of smoothers composing a trajectory generator subject to kinematic limits to guarantee the minimum duration of the trajectory;

- translate the design of a minimum duration rest-to-rest trajectory subject to any number of kinematic bounds into the solution of an optimization problem;

- merge kinematics and frequency specifications in such a way that the minimum complexity of the generator (in terms of the number of smoothers) and accordingly the minimum order of the output trajectory is always obtained;

- provide a MATLAB tool that computes the parameters of the optimal S-shaped trajectory and builds a SIMULINK block for the generation of the trajectory and its derivatives (in [24] the link to download this tool is reported).

Interesting enough, with this work a new trajectory design and optimization paradigm is proposed: while the most of planners available in the literature have a fixed structure, e.g. based on a parametric expression of the trajectory, and the free parameters are chosen using a nonlinear optimization procedure to comply with the given constraints [25], [26], according to a philosophy that can be defined top-down, in our approach the perspective can be defined bottom-up since each specification is translated in an element of the trajectory planner and then all these basic bricks are combined together in an optimal manner. The result of this design method is the shortest S-shaped trajectory compliant with all the specifications. Moreover, in most cases, this trajectory is the signal of the shortest duration, considering also signals obtained with other techniques, like e.g. input shaping. For this reason, although the proposed method leads to a trajectory planner that can be used online by providing to the chain of filters the desired target point, it also represents an efficient way for designing optimal trajectories for offline applications.

The paper is organized as follows. In Sec. III the problem of the generation of S-curve trajectories employing rectangular smoothers is summarized. In Sec. III the procedure for designing a trajectory planner that produces minimum-time trajectories under kinematic limits is presented, and then in Sec. IV the optimization procedure is further extended to take into account frequency constraints imposed by the suppression of residual vibrations. Experimental tests reported in Sec. V shows the effectiveness of the proposed approach. Concluding remarks are provided in the last section.

\section{Generation OF S-CURVE TRAJECTORIES ViA DYNAMIC FILTERS}

According to their standard definition, S-curve trajectories of order $n$ are trajectories composed of $2^{n}-1$ polynomial segments properly joined to assure the continuity of the derivative up to the order $n-1$. In [22] it has been shown that such a kind of trajectory can be generated by filtering a step

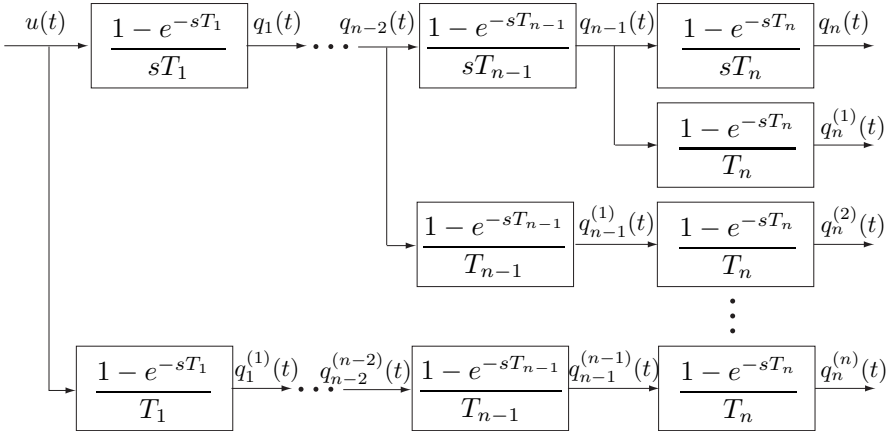

Fig. 1. Cascade of $n$ filters for the generation of multi-segment trajectories $q_{n}(t)$ of class $\mathcal{C}^{n-1}$ and all the derivatives $q_{n}^{(i)}(t), i=1, \ldots, n$.

input with a cascade of $n$ dynamic filters, called rectangular smoothers, characterized by the transfer function

$$
M_{i}(s)=\frac{1}{T_{i}} \frac{1-e^{-s T_{i}}}{s}
$$

where the parameter $T_{i}$ is a time length that determines the (finite) duration of the impulse response of each filter. Accordingly, the total duration of the trajectory produced by a step input is $T_{t o t}=\sum_{k=1}^{n} T_{k}$. In Fig. 1] the structure of the trajectory planner is shown. The input $u(t)$ is defined as

$$
u(t)=q_{i n}+q_{f i n} \cdot h(t)
$$

where $h(t)$ denotes the unit step function, while $q_{i n}$ and $q_{f i n}$ are initial and final position, respectively. Note that, besides the position signal, the cascade of filters also provides the derivatives of this signal that in many practical applications are necessary, e.g. for computing a feedforward control action. Moreover, it is worth noting that in modern motion control systems the generation of the trajectory at discrete-time instants can be obtained by simply considering in the scheme of Fig. 1 the discrete-time counterpart of (1), i.e.

$$
\begin{aligned}
M_{i}(z) & =\frac{1}{N_{i}} \frac{1-z^{-N_{i}}}{1-z^{-1}} \\
& =\frac{1}{N_{i}}\left(1+z^{-1}+z^{-2}+\cdots+z^{-\left(N_{i}-1\right)}\right) .
\end{aligned}
$$

where $N_{i}=\operatorname{ceil}\left(T_{i} / T_{s}\right)$, being $T_{s}$ the sampling time. The output of the trajectory planner is a symmetric S-shaped trajectory, that as well-known is composed of three different phases: acceleration, cruising at a constant velocity, deceleration. Acceleration and deceleration segments are composed of three different phases with a positive, null, and negative jerk. Each of the non-null jerk segments may be composed of three phases and so on, depending on the order of the trajectory. The duration of each phase is determined by the parameters $T_{i}$ of the filters as shown in Fig. 2 where the profiles of a 4 -th order trajectory are shown. In particular, each "impulse" composing a given derivative profile has a duration equal to $\sum_{k=i}^{n} T_{k}$ where $i$ denotes the order of the considered derivative.

\section{A. Kinematic constraints handling}

A noteworthy property of the trajectory planner illustrated in Fig. 1 is the possibility of imposing bounds on the trajectory derivatives by properly selecting the parameters $T_{i}$ 


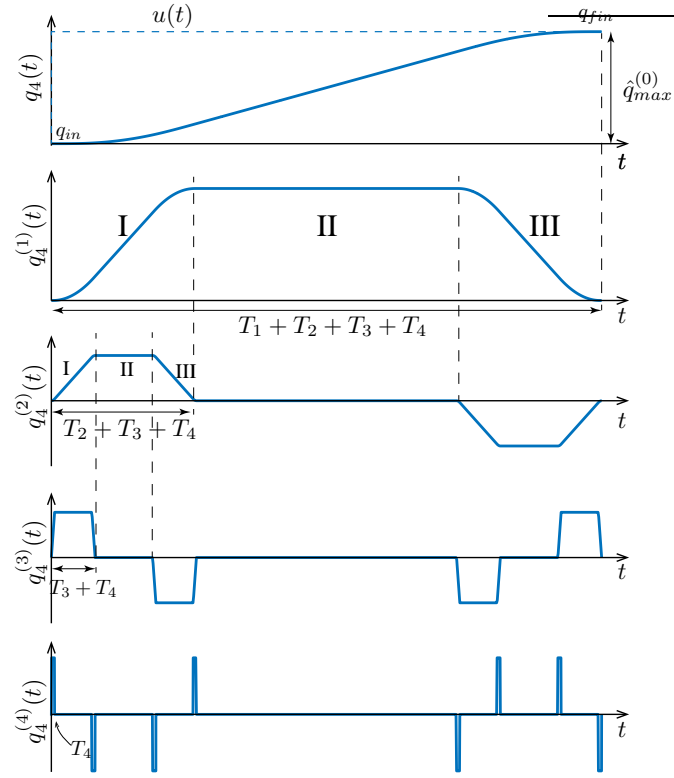

Fig. 2. Motion profiles of a 4-th order trajectory generated by a cascade of 4 rectangular smoothers.

of each smoother. In particular, by considering $n$ symmetric constraints, i.e. $\hat{q}_{\min }^{(i)}=-\hat{q}_{\max }^{(i)} i=1, \ldots, n$, it is possible to assure that

$$
-\hat{q}_{\max }^{(i)} \leq q^{(i)}(t) \leq \hat{q}_{\max }^{(i)}
$$

if a cascade of $n$ smoothers is adopted and their parameters $T_{i}$ are chosen as

$$
T_{i}=\frac{\hat{q}_{\max }^{(i-1)}}{\hat{q}_{\max }^{(i)}}, \quad i=1, \ldots, n
$$

where $\hat{q}_{\max }^{(0)}$ denotes the magnitude of required displacement or of the maximum displacement in case of generation of multiple trajectories, i.e. $\hat{q}_{\max }^{(0)}=\max _{i}\left|q_{f i n, i}-q_{i n, i}\right|$ being $q_{i n, i}$ and $q_{f i n, i}$ the start and the end position of the $i$-th trajectory segment composing the motion profile. For more details see [22], where the origin of (3) is explained.

\section{B. Residual vibration suppression}

The generation of a trajectory via smoothing filters allows to tackle the problem of the vibrations that the motion may cause in a resonant system, without the need for further actions like, for instance, the use of input shapers, which are a rather standard filtering technique for residual vibration suppression, see [17], [18], [19].

From a theoretical point of view, a rectangular smoother like (1) inserted in the trajectory planner of Fig. 2] leads to the complete suppression of the residual vibration caused by an undamped $(\delta=0)$ resonant mode at frequency $\hat{\omega}$, that may affect a mechanical system, if and only if the parameter $T_{i}$ of this smoother is a multiple of the period of this oscillation, i.e.

$$
T_{i}=k \frac{2 \pi}{\hat{\omega}}, \quad k=1,2, \ldots
$$

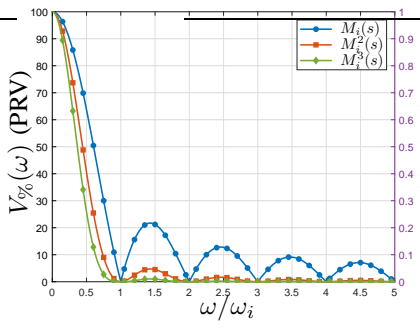

(a)

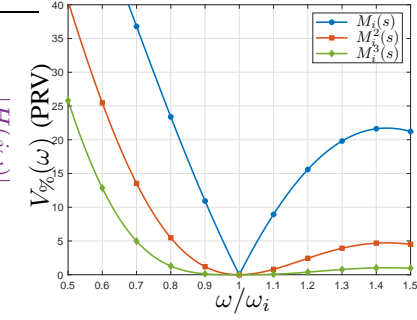

(b)
Fig. 3. Percentage Residual Vibration (PRV)/Frequency response of rectangular smoothers $M_{i}^{k}(s), k=1,2,3$ as a function of the normalized frequency $\omega / \omega_{i}$ (a) and magnification in the neighborhood of the first zero (b).

The proof is straightforward if the frequency response of the smoother is considered:

$$
M_{i}(j \omega)=\frac{1}{T_{i}} \frac{1-e^{j \omega T_{i}}}{j \omega}=e^{-j \frac{\omega T_{i}}{2}} \frac{\sin \left(\frac{\omega T_{i}}{2}\right)}{\frac{\omega T_{i}}{2}} .
$$

As shown in Fig. 3, the magnitude of the frequency response

$$
\left|M_{i}(j \omega)\right|=\left|\frac{\sin \left(\frac{\omega T_{i}}{2}\right)}{\frac{\omega T_{i}}{2}}\right|=\left|\operatorname{sinc}\left(\frac{\omega}{\omega_{i}}\right)\right|,
$$

where $\operatorname{sinc}(x)=\frac{\sin (\pi x)}{\pi x}$ denotes the normalized sinc function and $\omega_{i}=\frac{2 \pi}{T_{i}}$, is null for $\omega=k \omega_{i}, k=1,2 \ldots$ It is possible to prove that, except for the constant factor 100 , the frequency response of a generic filter coincides with the Percentage Residual Vibration 1 (PRV), which is defined as the ratio of the vibration with input filter to that without filter and is used to measure the capability of the filter to reduce the residual vibration as a function of the actual resonance frequency $\omega$ of the plant [27].

Therefore, it is straightforward to conclude that, in nominal conditions, the residual vibration at $\hat{\omega}$ is completely suppressed if and only if $\hat{\omega}=k \omega_{i}$, i.e.

$$
\hat{\omega}=k \frac{2 \pi}{T_{i}} \Longleftrightarrow T_{i}=k \frac{2 \pi}{\hat{\omega}}, \quad k=1,2, \ldots
$$

Obviously, the capability of the filter of effectively suppressing the vibration relies on the correct evaluation of $\hat{\omega}$. When the natural frequency $\omega$ of the plant differs from the nominal value $\hat{\omega}$ the capability of reducing the oscillation progressively lowers as the estimation error grows in magnitude and consequently the PRV increases, see Fig. 3 From this figure, it is possible to notice that this problem can be alleviated by considering more than one smoother for a specific frequency $\hat{\omega}$, reducing in this way the magnitude of the frequency response of the multiple smoothers around $\hat{\omega}$ and accordingly the related PRV, see Fig. 3(b). For instance, by assuming $\omega_{i}=\hat{\omega}$ and considering an error of $+10 \%$ in the estimation of the resonant frequency $\hat{\omega}$ (the actual frequency is $\omega=1.1 \hat{\omega}$ and therefore $\omega / \omega_{i}=1.1$ ), with a single smoother the PRV is $8.9 \%$. This means that the application of the smoother reduces the vibration at $\omega$ by $91.1 \%$. If two smoothers are considered the PRV becomes $0.8 \%$, while with a third smoother the PRV

\footnotetext{
${ }^{1} V_{\%}(\omega)=100|H(j \omega)|$, where $V_{\%}(\omega)$ denotes the PRV and $H(s)$ is the transfer function of the filter.
} 
is $0.1 \%$, with a reduction of $99.2 \%$ and $99.9 \%$ respectively. Finally, it is worth mentioning that if $\delta \neq 0$ the so-called exponential smoothers, that have been proposed in [28], should be considered instead of rectangular smoothers to assure a complete vibration suppression. However, it is possible to prove that if $\delta \leq 0.01$ the PVR caused by the assumption $\delta=0$, and accordingly by the use of rectangular smoothers, is smaller than $1 \%$. In general, the application of rectangular smothers, designed in accordance with (4), can effectively reduce the vibrations caused in poorly damped plants.

\section{OPTIMIZATION OF THE TRAJECTORY DURATION UNDER KINEMATIC CONSTRAINTS}

The condition (3) alone does not guarantee the minimum duration of the trajectory but only the compliance with the given kinematic constraints. To assure the time-optimality of the motion profile generated by the chain of smoothers the parameters $T_{i}$ found with (3) must comply with the further conditions

$$
T_{i} \geq \sum_{k=i+1}^{n} T_{k}, \quad i=1, \ldots, n-1 .
$$

These inequalities guarantee that all the kinematic bounds are reached by the derivatives of the computed trajectory. This means that there exists at leas 2 an interval in which $\left|q^{(i)}(t)\right|=$ $\hat{q}_{\text {max }}^{(i)}$ and $q^{(i+1)}(t)=q^{(i+2)}(t)=\cdots=q^{(n)}(t)=0$ for $i=$ $1, \ldots, n-1$. For instance, in Fig. 2 this phase is highlighted in the velocity and acceleration profiles where it is denoted with symbol II. The duration of this interval for a generic derivative of order $i$ can be computed as the difference between the duration of the impulses composing this derivative and twice the duration of the impulses composing the derivative of order $i+1$, i.e.

$$
d_{I I, i}=\sum_{k=i}^{n} T_{k}-2 \sum_{k=i+1}^{n} T_{k}
$$

Therefore, it is sufficient to impose that $d_{I I, i} \geq 0, i=$ $1, \ldots, n-1$ to deduce the inequalities (6). If all the parameters $T_{i}$ meets (6) the S-shaped trajectory is optimal in time since, as shown in Fig. 2, at each time $t$ at least a derivative is equal to the given bounds and (apparently) it is not possible to further reduce its duration without violating one of these limits. However, when the order of the trajectory is higher than 3, a new phenomenon appears that, in some cases, may lead to a reduction of the overall duration of the motion. If $d_{I I, i}=0$ the $i$-th derivative reaches the related limit, but only for a period of duration zero. In this case, the profile of the $(i+2)$-th derivative goes to zero from a given value and then again to this value, while the $(i+3)$-th derivative is characterized by two consecutive impulses with the same shape but opposite sign. The Figure 4 shows this situation when a 4-th order trajectory is considered and

$$
d_{I I, 1}=0 \Longleftrightarrow T_{1}=T_{2}+T_{3}+T_{4}
$$

\footnotetext{
${ }^{2}$ Because of the symmetry of the motion profiles there exist $2^{i-1}$ segments that meet the condition.
}

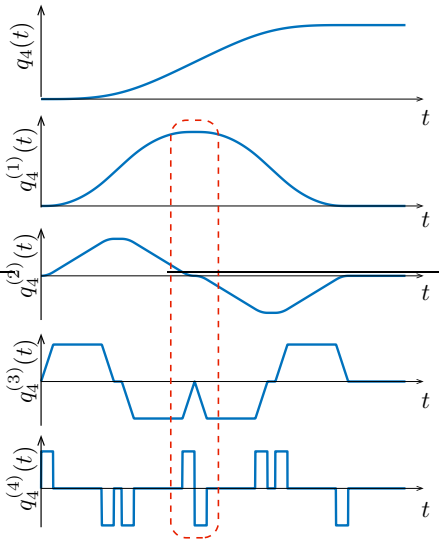

(a)

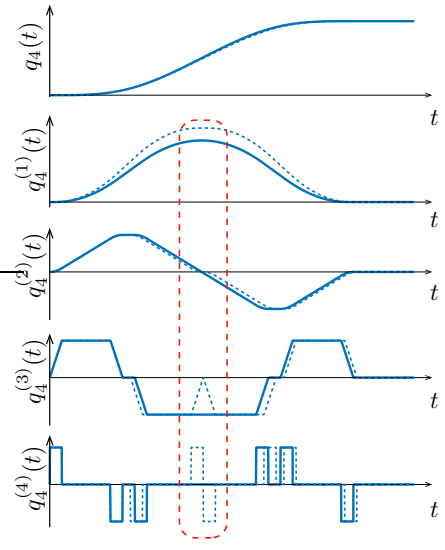

(b)
Fig. 4. Motion profiles of a 4-th order trajectory generated by a cascade of 4 rectangular smoothers with $d_{I I, i}=0 \Longleftrightarrow T_{1}=T_{2}+T_{3}+T_{4}$ (a) and with $T_{1}=T_{2}+T_{3}$ (b).

The velocity does not maintain a constant value but the acceleration phase is immediately followed by the deceleration phase. In this case, the jerk goes to zero from $q_{\min }^{(3)}$ and then it comes again to $q_{m i n}^{(3)}$. Therefore, it is possible to reduce the duration of the trajectory by overlapping acceleration and deceleration phases so that the jerk remains constant. Accordingly, the duration of the velocity profile, that coincides with the duration of the overall trajectory, must equal twice the duration of the acceleration period minus the duration of a snap impulse, i.e.

$$
\sum_{k=1}^{4} T_{k}=2 \sum_{k=2}^{4} T_{k}-T_{4}
$$

Note that this relation can be reduced to

$$
T_{1}=T_{2}+T_{3} .
$$

In this case, the value of $T_{1}$ does not meet the original condition (6). However, as shown in Fig. 4(b), the trajectory is still compliant with the initial bounds. By comparing the motion profiles obtained under the same limits it is possible to appreciate the effects caused by the computation of the parameter $T_{1}$ according to (9) instead of (8): the trajectory reaches the same limits except for the velocity, whose peak value decreases, and the total duration decreases as well. This suggests that the original condition (6) among parameters $T_{i}$ can be relaxed by assuming a limit duration of the impulses composing the $i$-th derivative profile equal to the double of the duration of the impulses of the $(i+1)$-th derivative minus the length of the impulses of the derivative of order $i+3$, i.e.

$$
\sum_{k=i}^{n} T_{k} \geq 2 \sum_{k=i+1}^{n} T_{k}-\sum_{k=i+3}^{n} T_{k}
$$

This relationship can be simplified as

$$
T_{i} \geq T_{i+1}+T_{i+2}, \quad i=1, \ldots, n-1 .
$$

where it is assumed $T_{n+1}=0$. Moreover, it can be rewritten in a compact form as

$$
\mathbf{A} \mathbf{T} \leq \mathbf{0}_{n}
$$


where $\mathbf{T}=\left[T_{1}, T_{2}, \ldots, T_{n}\right]^{T}$ denotes the vector obtained by collecting the parameters of all the filters composing the trajectory generator,

$$
\mathbf{A}=\left[\begin{array}{rrrrrrr}
-1 & 1 & 1 & 0 & \ldots & 0 \\
0 & -1 & 1 & 1 & \ldots & 0 \\
\vdots & & \ddots & & & \vdots \\
0 & \cdots & 0 & -1 & 1 & 1 \\
0 & \cdots & & 0 & -1 & 1 \\
0 & \cdots & & 0 & 0 & -1
\end{array}\right]
$$

and $\mathbf{0}_{n}$ is a vector composed of $n$ zeros.

If the values computed with (3) meet the conditions (10), or equivalently (11), the compliance of the trajectory with the given bounds and its minimum duration are assured and, therefore, these values can be directly used in the planner without further actions. Conversely, if the initial values of parameters $T_{i}$ do not meet (10), they must be modified, and the vector $\mathbf{T}^{\star}$ containing the optimal values can be found by solving a nonlinear optimization problem, i.e.

$$
\mathbf{T}^{\star}=\arg \min _{\mathbf{T}} \sum_{k=1}^{n} T_{k}
$$

aiming at minimizing the total duration of the motion, subject to the linear constraint (11) and to the nonlinear constraints

$$
\begin{aligned}
& \hat{q}_{\max }^{(n)} \prod_{k=1}^{n} T_{k}=\hat{q}_{\max }^{(0)} \\
& \hat{q}_{\max }^{(n)} \prod_{k=i+1}^{n} T_{k} \leq \hat{q}_{\max }^{(i)}, \quad i=1, \ldots, n-1 .
\end{aligned}
$$

Conditions (13) and (14) assures the given kinematic bounds are never exceeded by the trajectory derivatives. They are derived from (3), that in the case of modifications of the constants $T_{i}$ (supposed complaint with (11)) relates these new values with the peak values $q_{\max }^{(i)}$ actually reached by the trajectory derivatives, i.e.

$$
T_{i}=\frac{q_{\max }^{(i-1)}}{q_{\max }^{(i)}} \Leftrightarrow q_{\max }^{(i)} T_{i}=q_{\max }^{(i-1)}, \quad i=1, \ldots, n
$$

By iterating (15) the relation

$$
q_{\max }^{(i)}=q_{\max }^{(n)} \prod_{k=i+1}^{n} T_{k}
$$

is readily deduced. Finally, by imposing

$$
\begin{cases}q_{\max }^{(i)}=\hat{q}_{\max }^{(i)} & \text { for } i=0, n \\ q_{\max }^{(i)} \leq \hat{q}_{\max }^{(i)} & \text { otherwise }\end{cases}
$$

in (16) the conditions (14) descend. Note that the actual value $q_{\max }^{(0)}$ does not depend on the parameters $T_{i}$ but only on the magnitude of the input step, having the filters a unitary static gain. Therefore, its value can be selected by imposing that the input reference is composed by step functions having a maximum magnitude $\hat{q}_{\max }^{(0)}$. For what concerns the derivative of order $n$, in an optimal multi-segment trajectory it is composed of constant segments, whose magnitude must be always equal to the maximum allowed value, i.e. $q_{\max }^{(n)}=\hat{q}_{\max }^{(n)}$.
The solution to the optimization problem (12) is extremely efficient. By applying the interior-point method for nonlinear optimization which is available in Matlab, via the fmincon function, the problem on a standard laptop equipped with an Intel Core i7-7700HQ CPU can be solved in less than one second. For instance, by considering constraints with a random distribution in the range $\left[10^{-2}, 10^{2}\right]$, the average time for computing a solution in $10^{5}$ tests is $0.1023 \mathrm{~s}$ for $n=4$ and $0.1606 \mathrm{~s}$ for $n=5$. Unfortunately, the convergency of the algorithm to the global minimum is not guaranteed. For instance, for $n=4$ the optimal solution is found in $98.26 \%$ of cases, while for $n=5$ the right solution is obtained in $97.15 \%$ of cases. For this reason, even if the constrained optimization problem is of fundamental importance for understanding the process that leads to the minimization of the duration of trajectories subject to kinematic constraints, the recursive solution that we proposed in [23] can be preferred for applications. Note that in principle both the approaches should provide the same final result, i.e. the value of the parameters $T_{i}$ that implies the minimum duration of the motion, but the latter method assures that this result is always found.

\section{OPTIMIZATION OF MULTI-SEGMENT POLYNOMIAL TRAJECTORIES WITH KINEMATIC CONSTRAINTS AND VIBRATION SUPPRESSION CAPABILITIES}

In this section, frequency constraints due to the suppression of residual vibrations are also considered in the optimization problem.

Suppose that a motion application requires:

- the compliance with kinematic constraints on the first $n$ derivatives;

- the cancellation of residual vibrations due to $m$ oscillating modes, possibly with multiple smoothers to increase the insensitivity to estimation errors (or variations) of the resonance frequency (the multiplicity of each smoother is $n_{i}$ ).

In principle, a trajectory planner able to deal with the above specifications is characterized by an order/number of smoothers equal to

$$
n_{\text {tot }}=n+\sum_{i=1}^{m} n_{i}
$$

being $n_{i}$ is the number of smoothers used to suppress the residual vibration at a specific frequency. Consequently, the duration of the rest-to-rest trajectory produced by the planner is

$$
T_{\text {tot }}=\sum_{i=1}^{n} T_{i}+\sum_{i=1}^{m} \sum_{j=1}^{n_{i}} T_{\hat{\omega}_{i}, j}
$$

where the parameters $T_{i}$ are computed according to 3 while $T_{\hat{\omega}_{i}, j}$ are obtained from the corresponding frequency $\hat{\omega}_{i}, i=$ $1, \ldots, m$ by using (4), i.e.

$$
T_{\hat{\omega}_{i}, j}=k_{j} T_{\hat{\omega}_{i}}, \quad k_{j}=1,2, \ldots
$$

being

$$
T_{\hat{\omega}_{i}}=\frac{2 \pi}{\hat{\omega}_{i}}
$$


input : the vectors $\boldsymbol{T}=\left[T_{i}\right], i=1, \ldots, n$ and $\boldsymbol{T}_{\omega}=\left[T_{\hat{\omega}_{i}}\right], i=1, \ldots, m_{t o t}$

output: the vector $T^{\star}$ containing the parameters of the optimal trajectory generator

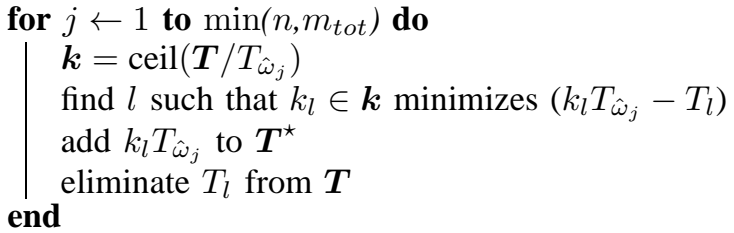

add to $\boldsymbol{T}^{\star}$ the remaining elements of $\boldsymbol{T}$ or $\boldsymbol{T}_{\omega}$

Algorithm 1: Algorithms for parameters optimization.

the period of the oscillation caused by the mode at $\hat{\omega}_{i}$. A superficial analysis would lead to the conclusion that the minimization of the total trajectory duration can be achieved by minimizing each term $T_{i}$ or $T_{\hat{\omega}_{i}, j}$. In particular, in 20, the value $k_{j}=1$ would be chosen since it corresponds to the shortest duration of every parameter $T_{\hat{\omega}_{i}, j}\left(=T_{\hat{\omega}_{i}}\right)$. This is exactly what has been done in our previous publication [23], where the additional consideration on the kinematic limitations imposed by the filters for vibrations suppression has been exploited to reduce the total number of required filters with respect to the initial value $n_{t o t}$. However, the devised procedure leads to a suboptimal result (in terms of number of smoothers and duration of the trajectory) that strongly depends on the specific input data (kinematic constraints and resonant frequencies). In many cases, the number $n_{t o t}$ of smoothers remains unchanged and accordingly, the total duration remains $T_{\text {tot }}$ computed according to (19).

Conversely, to always obtain the optimal trajectory, i.e. of minimal duration, it is necessary to use in (4) the degree of freedom offered by $k$. In this manner, for a given resonant frequency $\omega_{i}$ it is possible to substitute one of the parameters $T_{i}$, used for assuring the compliance with a kinematic bound, with the parameter $T_{\hat{\omega}_{i}, j}$ that better approximates this value from above. This procedure is made possible by the fact that vibration suppression at a specific frequency requires a parameter equal to or multiple of a prescribed value, while the compliance with the kinematic bounds is always guaranteed if parameters $T_{i}$ are larger in magnitude than the values initially computed with (3). This latter consideration is clear if the relationship

$$
q_{\max }^{(i)}=\frac{\hat{q}_{\max }^{(0)}}{\prod_{k=1}^{i} T_{k}}, \quad i=1, \ldots, n
$$

obtained from (15) is considered since it shows that an increase of any $T_{k}$ may produce only a reduction of the derivatives peak values. Remember that $\hat{q}_{\max }^{(0)}$ is a constant value related to the amplitude of the input signal.

The procedure for the optimization of the trajectory generator parameters is reported in the Algorithm 1 . The kinematic constraints are handled according to the procedure outlined in Sec. III and the vector $\boldsymbol{T}=\left[T_{i}\right], i=1, \ldots, n$ is built. For any resonant frequency $\hat{\omega}_{i}$, the fundamental time $T_{\hat{\omega}_{i}}$ defined in 21 is firstly computed. Then, these parameters are all collected in the vector $\boldsymbol{T}_{\omega}$ with the desired degree of multiplicity $n_{i}$. For instance, if the suppression of the vibration at $\hat{\omega}_{j}$ with a double filter is required, the vector $\boldsymbol{T}_{\omega}$ contains two times the element $T_{\hat{\omega}_{j}}$ and so on. Therefore, the total number of elements in $\boldsymbol{T}_{\omega}$ is $m_{t o t}=\sum_{i=1}^{m} n_{i}$.

According to the proposed method, for any element of the vector $\boldsymbol{T}_{\omega}$, related e.g. to the frequency $\hat{\omega}_{j}$, the new elements of $T^{\star}$

$$
T_{l}^{\star}=k_{l} T_{\hat{\omega}_{j}}
$$

is found that on the one side guarantees the suppression of the residual vibration at $\hat{\omega}_{j}$, being proportional to $T_{\hat{\omega}_{j}}$, and on the other hand approximates an element of $\boldsymbol{T}$. In particular, among all the components of $\boldsymbol{T}$ the one that minimizes the residual time

$$
\Delta T_{i}=k_{i} T_{\hat{\omega}_{j}}-T_{i}, \text { with } k_{i}=\operatorname{ceil}\left(\frac{T_{i}}{T_{\hat{\omega}_{j}}}\right)
$$

is selected. Suppose that the corresponding index is $l$. Note that the function ceil( $\cdot$ ) rounds its argument to the nearest integer towards infinity, therefore $k_{l} T_{\hat{\omega}_{j}} \geq T_{l}$. The value of $k_{l}$ is used in (23), while $T_{l}$ is eliminated from $\boldsymbol{T}$.

This operation is repeated until all the elements of $\boldsymbol{T}_{\omega}$ have been considered or vector $\boldsymbol{T}$ is empty. At the end of the procedure, residual elements of $\boldsymbol{T}_{\omega}$ or $\boldsymbol{T}$ are simply added to $T^{\star}$.

It is worth noting that for any pair of components of $\boldsymbol{T}$ and $\boldsymbol{T}_{\omega}$ only one element is added to $T^{\star}$. Accordingly, only one filter is sufficient to meet both the constraints and the overall number of smoothers for satisfying all the specifications becomes

$$
n_{\text {tot }}^{\star}=\max \left\{n, m_{\text {tot }}\right\}
$$

which is certainly smaller than the initial number $n_{t o t}$ reported in (18). Concerning the duration of the trajectory, the delay $T_{\hat{\omega}_{j}}+T_{i}$ caused by a pair of smoothers is replaced by $k_{i} T_{\hat{\omega}_{j}}$ with a consequent time reduction equal to

$$
\left(T_{\hat{\omega}_{j}}+T_{i}\right)-k_{i} T_{\hat{\omega}_{j}}=T_{\hat{\omega}_{j}}-\Delta T_{i}
$$

where $\Delta T_{i}$ is defined in (24). Since, according to this definition, the following bounds hold:

$$
0 \leq \Delta T_{i}<T_{\hat{\omega}_{j}}
$$

the reduction of the trajectory duration due to the fulfilment of two different specifications with a unique smoother is always strictly positive. In other words, the devised procedure always allows saving time. The minimization of $\Delta T_{i}$ has the purpose to maximize the reduction of the trajectory duration with respect to the one generated by the trivial cascade of $n_{t o t}$ smoothers, i.e. one for each specification. From a different perspective, the quantity

$$
\Delta T_{\text {tot }}=\sum_{i=1}^{n_{\text {tot }}^{\star}} \Delta T_{i}
$$

where $\Delta T_{i}$ is defined in (24) for $i \leq n$ and $\Delta T_{i}=T_{\hat{\omega}_{i}}$ otherwise, represents the total increment of the duration with 


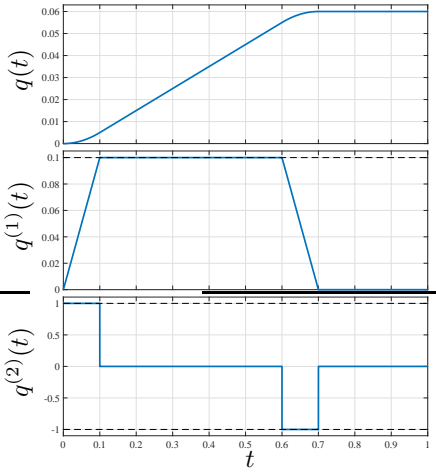

(a)

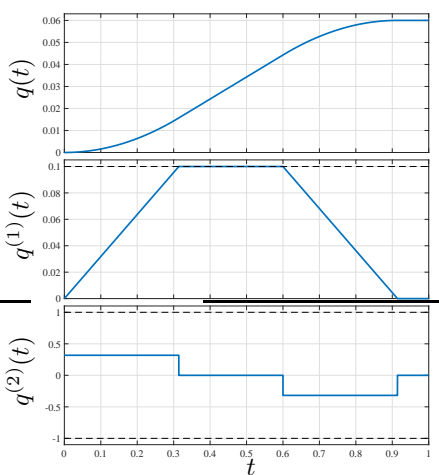

(c)

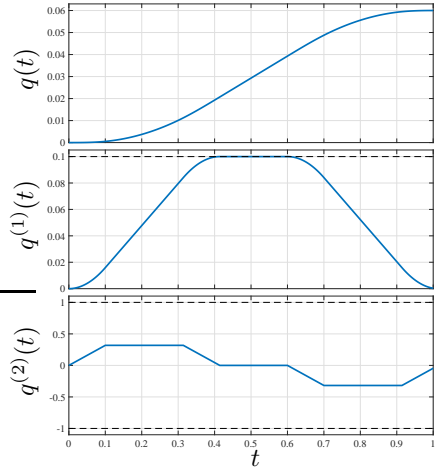

(b)

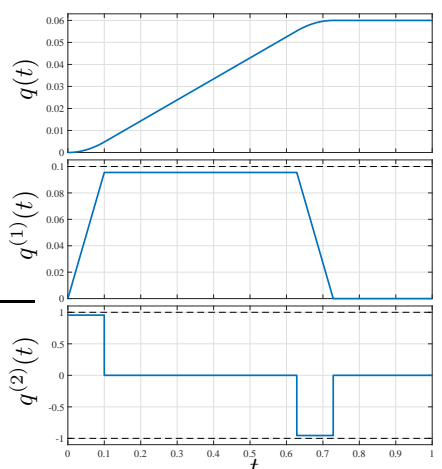

(d)
Fig. 5. Profiles of position, velocity and acceleration of produced by the trajectory planner that assures the compliance with the kinematic constraints 27 (a), a third order filter taking into account also the suppression vibration at $\hat{\omega}_{1}$ (b), the trajectory planner optimized as in [23 (c), and the trajectory planner optimized according the new procedure $(\mathrm{d})$.

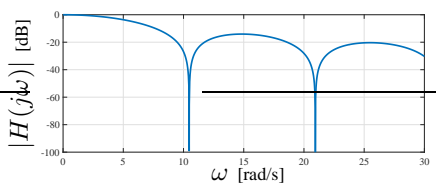

(a)

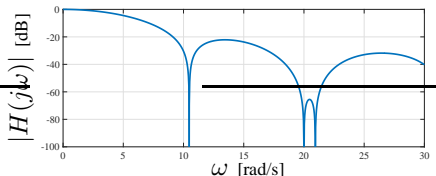

(c)

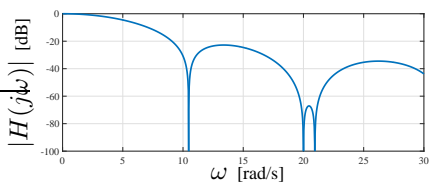

(b)

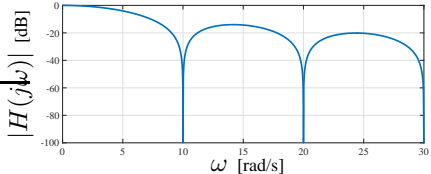

(d)
Fig. 6. Frequency response of the filters generating the trajectory profiles in Fig. 5

respect to the minimum-time trajectory defined by taking into account only the $n$ kinematic constraints. Accordingly the minimization of $\Delta T_{i}$ aims at minimizing the effects of residual vibrations suppression specifications with respect to the trajectory initially optimized only on the basis of kinematic constraints.

\section{A. A numerical example}

In order to exemplify how the proposed method works a simple numerical case is considered. In this example, the following specifications have been assumed:

$$
\hat{q}_{\max }^{(0)}=0.06 \mathrm{~m}, \quad \hat{q}_{\max }^{(1)}=0.1 \mathrm{~m} / \mathrm{s}, \quad \hat{q}_{\max }^{(2)}=1 \mathrm{~m} / \mathrm{s}^{2} .
$$

Accordingly, the parameters of the two smoothers for the generation of the minimum-time trajectory compliant with the given constraints computed according to the technique illustrated in Sec. III, are $T_{1}=0.6 \mathrm{~s}, T_{2}=0.1 \mathrm{~s}\left(T_{\text {tot }}=0.7\right.$ $\mathrm{s})$. The related motion profiles are shown in Fig. 5 (a). If now the suppression of the residual vibration at $\hat{\omega}_{1}=20 \mathrm{rad} / \mathrm{s}$ is required, the trajectory generator can be modified with the insertion of an additional smoother characterized by

$$
T_{\hat{\omega}_{1}}=\frac{2 \pi}{20}=0.3142 \mathrm{~s}
$$

The resulting profiles are reported in Fig. 5(b): the order of the trajectory increases of one and accordingly the acceleration becomes a continuous signal but, on the other hand, the maximum value the acceleration is considerably smaller than the desired bound and the duration of the trajectory becomes much longer $\left(T_{t o t}=1.0142 \mathrm{~s},+44.89 \%\right)$. As highlighted in Fig. 6(a)-(b), the frequency response of the initial second-order trajectory generator is modified by the additional smoother so that its magnitude becomes zeros at $\hat{\omega}_{1}$. A similar result is achieved with the approach previously proposed in [23], that simply replaces $T_{2}$ with $T_{\hat{\omega}_{1}}$. By comparing Figures 6(b) and (c) it is evident that although the trajectory generator is composed of two smoothers its frequency response is very similar to the one of the third-order filter (with a zero located exactly in $\left.\hat{\omega}_{1}\right)$. The total duration, however, is reduced as shown in Fig. 5(c). Note that in this case $T_{t o t}=0.9142 \mathrm{~s}$, i.e. $30.60 \%$ more than the original trajectory. Finally, considering the optimal method presented in this section the parameters $T_{1}$ of the original trajectory is replaced by $2 T_{\hat{\omega}_{1}}$. In this manner, the motion profiles are slightly modified with respect to the ones generated by the initial filter and do not reach the kinematic bounds, see Fig. 5(c), but the magnitude of the frequency response becomes null at $\hat{\omega}_{1}$ and therefore the residual vibration suppression is assured. The increase of the trajectory duration is modest, being $T_{t o t}=0.7283 \mathrm{~s}$ $\left(\Delta T_{\text {tot }}=0.0283 \mathrm{~s},+4.04 \%\right)$.

Interestingly enough, since the number of kinematic constraints is $n=2$, while the frequency specifications are $m_{t o t}=$ 1 , it is possible to improve the insensitivity of the trajectory generator in vibration suppression without increasing its order by simply considering two frequency constraints at $\hat{\omega}_{1}$. In this case, $T_{1}^{\star}=0.6283 \mathrm{~s}$ and $T_{2}^{\star}=0.3142 \mathrm{~s}$. With these parameters, the frequency response of the trajectory generator assumes the shape reported in Fig. 7 (a), from which it is clear that the reduction of the vibrations around the frequency $\hat{\omega}_{1}$ is strongly improved in term of sensitivity with respect to estimation errors/variations of $\hat{\omega}_{1}$. Note that, in the ideal case, the produced second-order trajectory is able to suppress the residual vibration at $\hat{\omega}_{1}$ even if the jerk is boundless.

Finally, if the plant is characterized by a second mode of vibration at $\hat{\omega}_{2}=25 \mathrm{rad} / \mathrm{s}$, the devised procedure can effectively reduce the vibrations of both the modes by collocating two zeros at $\hat{\omega}_{1}$ and $\hat{\omega}_{2}$ respectively, see Fig. 7b). In this case, the trajectory planner is composed by two smoothers with $T_{1}^{\star}=2 T_{\hat{\omega}_{1}}=0.6283 \mathrm{~s}$ and $T_{2}^{\star}=T_{\hat{\omega}_{2}}=0.2513 \mathrm{~s}$. 


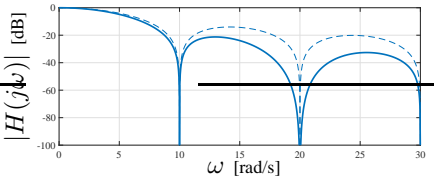

(a)

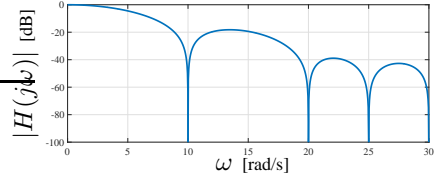

(b)
Fig. 7. Frequency response of a trajectory planner designed according to the proposed procedure when a second-order vibration suppression at $\hat{\omega}_{1}$ is required (a) and when two different modes of vibrating must be suppressed (b). In the plot (a) the frequency response of the planner is compared with the frequency response obtained with a single frequency (thin dashed line), which is reported in Fig. 6) d).

\section{B. Remarks on the time-optimality of the trajectory}

The S-shaped trajectory obtained considering $n$ kinematic constraints and a single resonant frequency $\hat{\omega}_{1}$, possibly with a multiplicity higher than one, is time-optimal since it is generated with the minimum number of filters and each of these filters is characterized by the minimum value of the parameter $T_{i}^{\star}\left(=T_{i}+\Delta T_{i}\right)$ that allows meeting the constraints. Note that in this case, even if the minimization of $\Delta T_{i}$ is performed for each element of $\boldsymbol{T}_{\omega}$, considered in the given sequence, and it is not the result of a global optimization process that takes into account all the terms, the global minimum of $\Delta T_{t o t}$ will be found since all the components of $\boldsymbol{T}_{\boldsymbol{\omega}}$ are equal (being related to the same frequency). When several resonant frequencies are considered the ordered minimization of $\Delta T_{i}$, may not lead to the global minimum of $\Delta T_{t o t}$. However, since the values in $\boldsymbol{T}_{\omega}$ are considered in descending order (that is for ascending magnitude of the frequencies $\hat{\omega}_{j}$ ), the increments $\Delta T_{i}$ related to the values $\hat{\omega}_{j}$, which as shown in 26) is limited by $T_{\hat{\omega}_{j}}$, tend to be smaller and smaller, and accordingly the difference between a possibly suboptimal value of $\Delta T_{t o t}$ and the optimal one is certainly very small.

Provided that the trajectory obtained with the proposed method is the shortest one among all the possible S-shaped trajectories that meet the given specifications (at least for a single resonant frequency), the last question that arises concerns whether the proposed approach produces the optimal solution among all the possible motion profiles or it is possible to find alternative solutions that lead to shorter motions. The suppression of residual vibrations is generally treated as a filtering problem. In particular, input shapers are usually considered [17], [18], [19]. Within this broad family, Zero Vibration (ZV) input shapers involve the smallest additional delay and guarantee the compliance of the output signal with the constraints originally met by the input trajectory. If for a given resonance frequency $\hat{\omega}_{1}$ a ZV input shaper is considered, the additional delay with respect to the minimum time trajectory taking into account only kinematic constraints is $T_{z v}=\frac{\pi}{\hat{\omega}_{1}}$, i.e. half of the maximum theoretical additional duration $\Delta T_{i}$ produced by a smoother. However, $\Delta T_{i}$ is not fixed but is a stochastic value that depends on the constraints and has a uniform distribution over the interval $\left[0, \frac{2 \pi}{\hat{\omega}_{1}}\right)$. Therefore, for a generic $i=1, \ldots n$ the probability that the proposed method outperforms input shaping is

$$
\mathrm{P}\left(\Delta T_{i} \leq T_{z v}\right)=0.5
$$

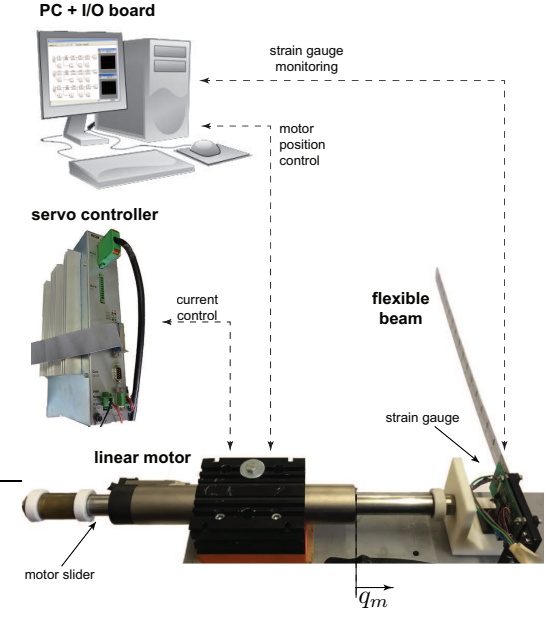

Fig. 8. Experimental setup based on a flexible link.

Since the algorithm selects the element that minimizes $\Delta T_{i}$, the probability that the increase in duration does not exceed $T_{z v}$ becomes

$$
\mathrm{P}\left(\bigcup_{i=1}^{n}\left(\Delta T_{i} \leq T_{z v}\right)\right)=1-0.5^{n}
$$

where $n$ is the number of kinematic constraints. Accordingly, for a second-order trajectory the probability of obtaining a shorter duration with the proposed method taking into account a single critical frequency is 0.75 , while for a third-order trajectory is 0.875 . When several (or multiple) frequency constraints are considered, formula (28) is not valid anymore, but in any case, this probability is never smaller than 0.5 . We can finally conclude that the proposed methods generally outperforms all existing solutions.

\section{EXPERIMENTAL VALIDATION OF THE OPTIMIZATION PROCEDURE OF TRAJECTORIES WITH KINEMATIC AND FREQUENCY CONSTRAINTS}

To validate the proposed method the same experimental setup as in [23] has been used. As shown in Fig. 8, the test-bed is based on a thin stainless steel flexible link directly connected to the slider of a linear motor. The side of the link connected to the motor is instrumented with a strain gauge that detects the local deformation of the beam. The link was originally part of the Rotary Flexible Link setup by Quanser ${ }^{\circledR}[29]$. Note that this setup is a typical test bed for residual vibration suppression [30], [31], but is has been also considered as a paradigmatic example of mechanical systems characterized by structural flexibilities that cause the so-called post-actuator modes [14] and which are quite common in the industrial practice.

The bending deformation of the link subject to the lateral acceleration $\ddot{q}_{m}$ of the motor, and detected by the strain gauge, is characterized by two modes located at $\hat{\omega}_{1} \approx 20.18 \mathrm{rad} / \mathrm{s}$ and $\hat{\omega}_{2} \approx 127.5 \mathrm{rad} / \mathrm{s}$, respectively. For more details about the system model refer to [23]. The damping coefficient of the first mode is $\delta=0.0043$, and therefore the filters can be designed with the assumption $\delta \approx 0$ that, as discussed in Sec. I-B, should assure a very low level of the residual vibration. To 
quantify the level of vibrations, the output voltage $V_{w}(t)$ of the strain gauge, which is proportional to the bending deformation, is directly used.

The goal of the experiments is to impose on the motor a prescribed motion minimizing the oscillations of the beam. In other terms, the tip of the link must follow the motor/base position as close as possible, in particular when the motion stops. This aim and even the (lumped-parameter) model of the system is very similar to that of a generic industrial positioning system where the load is driven by the actuator through a mechanical transmission affected by some elasticity, see [14]. Additionally, to cope with the physical limitations of the actuator the kinematic bounds (and the displacement) reported in (27), which have been already considered in [23], have been used. Then, to highlight the advantages of the proposed optimization technique, different approaches for the suppression of residual vibrations, based on smoothers and/or input shapers, have been compared with the minimum-time trajectory. Obviously, all the methods taken into account guarantee the compliance of the final motion profile with the initial kinematic constraints. In particular, Figure 9 shows the voltage signal $V_{w}(t)$ consequent to the application of the trajectories obtained with the following methods:

(a) Cascade of two smoothers that generates the minimumtime trajectory compliant with the given constraints. The parameters of the smoothers, computed in Sec. IV-A, are $T_{1}=0.6 \mathrm{~s}$ and $T_{2}=0.1 \mathrm{~s}\left(T_{\text {tot }}=0.7 \mathrm{~s}\right)$.

(b) Trajectory generator obtained with the approach here proposed by considering a first-order reduction of the vibration at $\omega_{r 1}$. The order of the generator remains 2 , while the parameters become $T_{1}=2 T_{\hat{\omega}_{1}}=0.6227$ $\mathrm{s}$, being $T_{\hat{\omega}_{1}}=\frac{2 \pi}{\hat{\omega}_{1}}=0.3114 \mathrm{~s}$, and $T_{2}=0.1 \mathrm{~s}$ $\left(T_{\text {tot }}=0.7227 \mathrm{~s}\right)$.

(c) Trajectory generator defined in (a) followed by a ZV input shaper with $T_{z v}=\frac{\pi}{\hat{\omega}_{1}}=0.1557 \mathrm{~s}\left(T_{t o t}=0.8557 \mathrm{~s}\right)$.

(d) Cascade of smoothers optimized according to the procedure proposed in [23] that leads to values $T_{1}=0.6 \mathrm{~s}$ and $T_{2}=T_{\hat{\omega}_{1}}=0.3114 \mathrm{~s}\left(T_{\text {tot }}=0.9114 \mathrm{~s}\right)$.

(e) Trajectory generator obtained with the approach here proposed by considering a second-order reduction of the vibration at $\hat{\omega}_{1}$. The parameters of the cascade of smoothers are $T_{1}=2 T_{\hat{\omega}_{1}}=0.6227 \mathrm{~s}$ and $T_{2}=T_{\hat{\omega}_{1}}=$ $0.3114 \mathrm{~s}\left(T_{\text {tot }}=0.9341 \mathrm{~s}\right)$.

(f) Trajectory produced by the generator defined in (a) filtered by an additional smoother for vibration suppression with $T_{3}=T_{\hat{\omega}_{1}}=0.3114 \mathrm{~s}\left(T_{\text {tot }}=1.0142 \mathrm{~s}\right)$.

(g) Trajectory generator obtained with the approach here proposed by considering a third-order reduction of the vibration at $\hat{\omega}_{1}$. The smoothers in the chain are three with parameters $T_{1}=2 T_{\hat{\omega}_{1}}=0.6227 \mathrm{~s}$ and $T_{2}=T_{3}=$ $T_{\hat{\omega}_{1}}=0.3114 \mathrm{~s}\left(T_{\text {tot }}=1.2454 \mathrm{~s}\right)$.

Note that, even if with a little difference in the frequency value $\hat{\omega}_{1}$, the cases (a)-(b)-(d)-(f) have been already analyzed in the numerical example of Sec. IV-A, where the corresponding motion profiles are shown. The trajectory obtained with the planner (f) is a standard third-order S-curve optimized for vibration suppression at the specific frequency $\hat{\omega}_{1}$. This
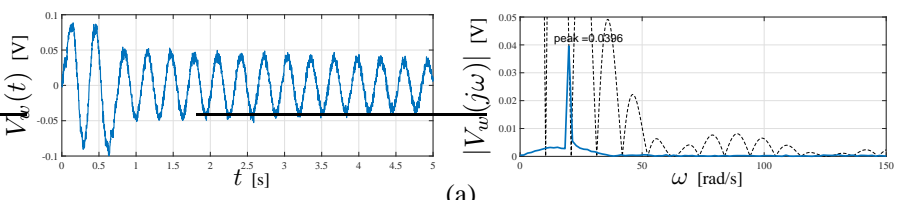

(a)
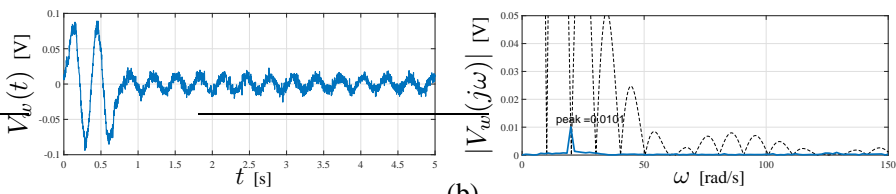

(b)
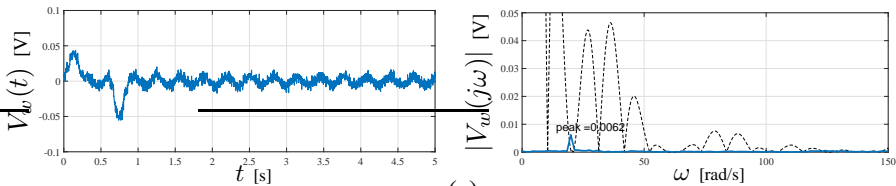

(c)
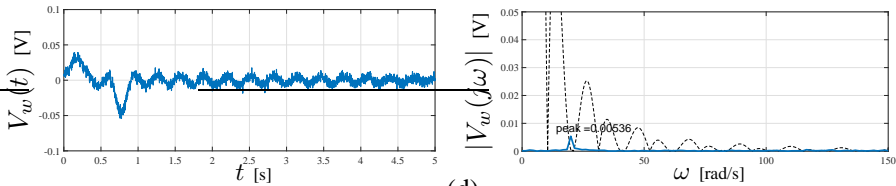

(d)
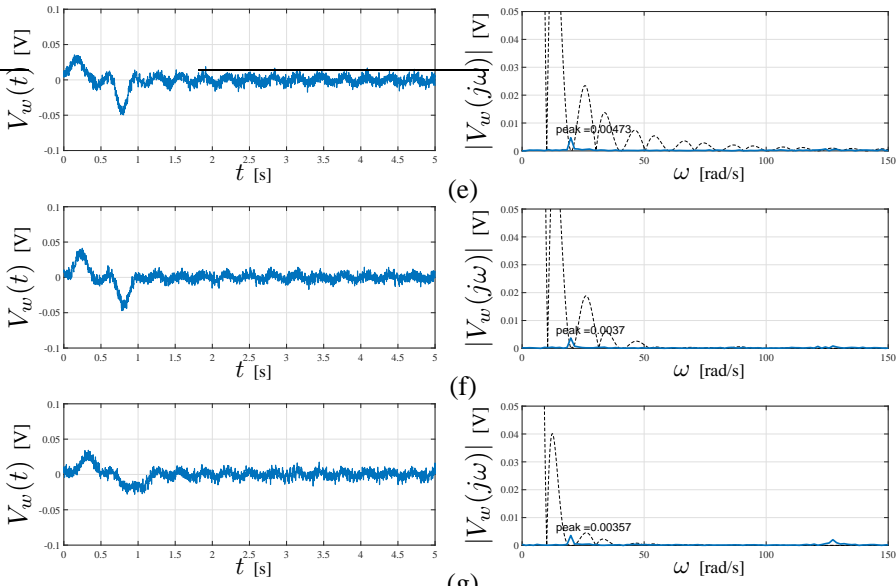

(f)

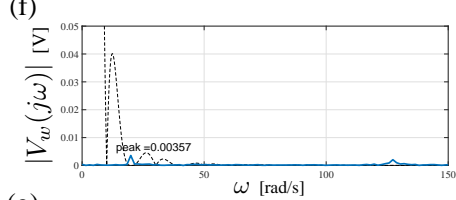

(g)

Fig. 9. Strain gauge output signals $V_{w}(t)$, and related spectrum $\left|V_{w}(j \omega)\right|$, produced by different motion profiles applied to the flexible link: trapezoidal velocity trajectory compliant with kinematic constraints on velocity and acceleration (a), same trajectory optimized for residual vibration suppression according to the method here proposed (b), initial trapezoidal trajectory filtered by a ZV input shaper (c), trajectory optimized for residual vibration suppression according to the method proposed in [23] (d), trajectory optimized for residual vibration suppression with a second- and a third-order smoother (e) and (g) respectively and initial trajectory, compliant with the kinematic limits, filtered by an additional smoother for vibrations suppression (f).

type of planner obtained by simply gathering the smoothers related to the different specifications (bounds on velocity, acceleration and vibration suppression), without a real integration/optimization, corresponds to the initial approach proposed in [22]. Note that this solution also coincides with the trajectory proposed in [21], where FIR filter is applied to a trapezoidal velocity, defined analytically.

The goal of this experimental activity is to measure the actual capability of the considered profiles in reducing the residual vibrations. For this reason, the signal $V_{w}(t)$, which is affected by a high level of noise, has been analyzed in the frequency domain, by applying a Fast Fourier Transform (FFT) to the 


\begin{tabular}{l|llll}
\hline & $T_{\text {tot }}[\mathrm{s}]$ & $\Delta T_{\text {tot }} \%$ & $V[\mathrm{mV}]$ & $\Delta V \%$ \\
\hline (a) & 0.7 & 0.00 & 39.6 & 0.00 \\
(b) & 0.7227 & +3.24 & 10.1 & -74.49 \\
(c) & 0.8557 & +22.24 & 6.2 & -84.34 \\
(d) & 0.9114 & +30.20 & 5.05 & -87.24 \\
(e) & 0.9341 & +33.44 & 4.81 & -87.85 \\
(f) & 1.0142 & +44.88 & 3.82 & -90.35 \\
(g) & 1.2454 & +77.91 & 3.7 & -90.65 \\
\hline
\end{tabular}

TABLE I

OVERALL DURATION $T_{t o t}$ OF THE DIFFERENT TRAJECTORIES CONSIDERED IN THE EXPERIMENTAL TESTS AND RELATED RESIDUAL VIBRATION LEVEL $V$ : ABSOLUTE VALUES AND PERCENTAGE VARIATIONS WITH RESPECT TO THE MINIMUM-TIME TRAJECTORY UNDER KINEMATIC CONSTRAINTS.

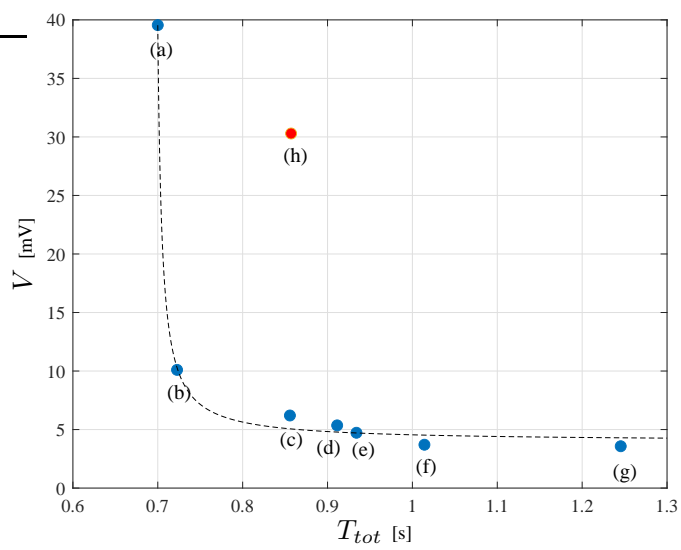

Fig. 10. Residual vibration level as a function of the trajectory duration of the different trajectories considered in the experimental tests. The point (h) in the map represents a standard S-curve trajectory not optimized for vibration suppression but compliant with the given kinematic limits.

samples of $V_{w}(t)$ measured after the reference motion stops. The spectra $\left|V_{w}(j \omega)\right|$ caused by the trajectories considered in the comparison are shown in Fig. 9 next to the corresponding profile (in the same figure the frequency spectrum of the trajectory generator is reported with a black dashed line). Note that this type of analysis allows to evaluate even small differences in signals that in some cases are very similar, see e.g the cases (c)-(f). All the spectra exhibit a peak value at the frequency $\hat{\omega}_{1}$, whose magnitude $V$ represents a good estimation of the residual vibration level. The contribution of the second mode at $\hat{\omega}_{2}$ is practically not appreciable, because of the low-pass characteristic of all the trajectory generators based on smoothers.

The results of the experiments are summarized in Tab. [ while a pictorial representation is reported in Figure 10, which contains a map of the pairs trajectory duration-vibration level $\left(T_{t o t}-V\right)$ for all the considered motion profiles. It is clear that the trajectory generator designed with the proposed approach - case (b) - provides the shortest trajectory able to effectively reduce the residual vibration. With a very modest increase of the duration with respect to the optimal trajectory under the kinematic constraints $(+3.24 \%)$ the new motion profile drastically reduces the level of vibration $(-74.49 \%)$. As mentioned in previous sections, by increasing the order of insensitivity at the critical frequency $\hat{\omega}_{1}$, i.e. the number of smoothers with null frequency response at this value, it is possible to further enhance the capability of lowering the level of the vibrations at the price of longer durations, see cases (e) and (g). Alternative approaches like the application of a ZV input shaper to the original trajectory - case (c) - or the optimization proposed in [23] - case (d) - lead to intermediate results in terms of duration and vibration level even if it is worth highlighting that the introduced delays are definitely higher than the approach proposed in this paper while the gain in terms of vibration amplitude is very little. The third-order S-curve trajectory (f) behaves similarly, since it causes a notable reduction of the vibration amplitude but at the price of a very large additional delay. It is worth noticing that such a kind of curve, even if characterized by a limited jerk, is not able to suppress residual vibrations when it is not properly designed: the point (h) in the map is obtained by considering the trajectory (a) filtered by an additional smoother with $T_{3}=T_{\hat{\omega}_{1}} / 2$. In this case, the duration is the same obtained with the ZV input shaper (c) but the magnitude of the residual vibration is much larger, even if the trajectory has a limited jerk.

The points reported on the map with blue color, representing the trajectories optimized for vibration suppression, can be effectively approximated using a rectangular hyperbola, that provides general hints on the problem of vibrations reduction: the vibration level is inversely proportional to the duration of the imposed motion, however, the level of vibrations rapidly tends to an asymptotic value. Note that this value is not zero since, besides an estimation error on the value of $\hat{\omega}_{1}$, the plant is characterized by nonlinear phenomena. Clearly, the case that optimizes both the criteria (duration and vibration magnitude) corresponds to the closest point to the origin, which is the trajectory (b) obtained with the procedure proposed here.

The use of several smoothers for increasing the insensitivity to a specific resonant mode may be not useful since it implies a notable increase in the duration with marginal improvements on the vibration level. The experiments prove that the limitation of the jerk is neither sufficient nor necessary to achieve vibration suppression, but a proper design of the trajectory generator can lead to very good results even in case of a second-order trajectory. In general, the best trade-off between trajectory duration and capability of reducing vibrations is obtained when the insensitivity of the trajectory planner is equal to the number of kinematic constraints, minimizing in this way the final order of the planned trajectory.

\section{Conclusions}

In this paper, a novel procedure for the design of an Sshaped trajectory planner able to deal with kinematic constraints and vibration suppression issues is proposed. The method is based on a combination of filters, that in the literature are well-known with the name of rectangular smoothers, and consists of an algorithm that, starting from the desired specifications, provides the number and the parameters values of these filters that guarantee the minimum duration of the generated motion profile. The obtained trajectory is optimal, i.e. it is not possible to find a different trajectory compliant with the same constraints which is shorter in time. Additionally, the 
order of the trajectory is not fixed a priori but depends on the number of specifications and, in principle, can be very high. Nevertheless, the proposed approach leads to the minimum possible order. Experiments on a test-bed based on a flexible link show both the effectiveness of the proposed method and the very simple and computationally efficient structure of the planner.

Future work will deal with the problem of adapting the trajectory generated with the smoothers in order to optimize the residual vibrations suppression for plants characterized by varying resonant modes, and more in general, with the problem of taking into account constraints that can change over time.

\section{REFERENCES}

[1] K. Erkorkmaz and Y. Altintas, "High speed cnc system design. part i: Jerk limited trajectory generation and quintic spline interpolation," vol. 41, pp. 1323-1345, 072001.

[2] P. Lambrechts, M. Boerlage, and M. Steinbuch, "Trajectory planning and feedforward design for electromechanical motion systems," Control Engineering Practice, vol. 13, pp. 145-157, 2005.

[3] T. Kroger, A. Tomiczek, and F. M. Wahl, "Towards on-line trajectory computation," in 2006 IEEE/RSJ International Conference on Intelligent Robots and Systems, Oct 2006, pp. 736-741.

[4] T. Kroger and F. M. Wahl, "Online trajectory generation: Basic concepts for instantaneous reactions to unforeseen events," IEEE Transactions on Robotics, vol. 26, no. 1, pp. 94-111, Feb 2010.

[5] K. D. Nguyen, T.-C. Ng, and I.-M. Chen, "On algorithms for planning s-curve motion profiles," International Journal of Advanced Robotic Systems, vol. 5, no. 1, p. 11, 2008.

[6] K. L. Knierim and O. Sawodny, "Real-time trajectory generation for three-times continuous trajectories," in 2012 7th IEEE Conference on Industrial Electronics and Applications (ICIEA), July 2012, pp. 1462 1467.

[7] B. Ezair, T. Tassa, and Z. Shiller, "Planning high order trajectories with general initial and final conditions and asymmetric bounds," The International Journal of Robotics Research, vol. 33, no. 6, pp. 898-916, 2014.

[8] R. Zanasi, C. G. L. Bianco, and A. Tonielli, "Nonlinear filter for the generation of smooth trajectories," Automatica, vol. 36, pp. 439-448, 2000.

[9] C. Zheng, Y. Su, and P. Muller, "Simple online smooth trajectory generations for industrial systems," Mechatronics, vol. 19, pp. 571-576, 2009.

[10] L. Biagiotti and R. Zanasi, "Time-optimal regulation of a chain of integrators with saturated input and internal variables: an application to trajectory planning," IFAC Proceedings Volumes, vol. 43, no. 14, 2010.

[11] C. G. L. Bianco and F. Ghilardelli, "A discrete-time filter for the generation of signals with asymmetric and variable bounds on velocity, acceleration, and jerk," IEEE Transactions on Industrial Electronics, vol. 61, no. 8, pp. 4115-4125, Aug 2014.

[12] A. Gasparetto and V. Zanotto, "A technique for time-jerk optimal planning of robot trajectories," Robotics and Computer-Integrated Manufacturing, vol. 24, no. 3, pp. 415 - 426, 2008.

[13] A. Olabi, R. Béarée, O. Gibaru, and M. Damak, "Feedrate planning for machining with industrial six-axis robots," Control Engineering Practice, vol. 18, no. 5, pp. 471-482, 2010.

[14] P.-J. Barre, R. Béarée, P. Borne, and E. Dumetz, "Influence of a jerk controlled movement law on the vibratory behaviour of high-dynamics systems," Journal of Intelligent and Robotic Systems, vol. 42, pp. 275293, 2005.

[15] C. Ha, K. Rew, and K. Kim, "Formulating a laplace domain approach for tuning motion profiles," in 2011 IEEE/ASME International Conference on Advanced Intelligent Mechatronics (AIM), 2011, pp. 356-360.

[16] D. Lee and C. Ha, "Optimization process for polynomial motion profiles to achieve fast movement with low vibration," IEEE Transactions on Control Systems Technology, vol. 28, no. 5, pp. 1892-1901, 2020.

[17] N. C. Singer and W. P. Seering, "Preshaping command inputs to reduce system vibration," ASME Journal of Dynamic Systems, Measurement, and Control, vol. 112, pp. 76-82, 1990.
[18] W. Singhose, N. Singer, and W. Seering, "Comparison of command shaping methods for reducing residual vibration," in Third European Control Conf, 1995, pp. 1126-1131.

[19] N. Singer, W. Singhose, and W. Seering, "Comparison of filtering methods for reducing residual vibration," European Journal of Control, vol. 5, pp. 208-218, 1999.

[20] M. O. T. Cole and T. Wongratanaphisan, "A direct method of adaptive fir input shaping for motion control with zero residual vibration," IEEE/ASME Transactions on Mechatronics, vol. 18, no. 1, pp. 316-327, 2013.

[21] P. Besset and R. Bearee, "Fir filter-based online jerk-constrained trajectory generation," Control Engineering Practice, vol. 66, pp. 169 - 180, 2017.

[22] L. Biagiotti and C. Melchiorri, "Fir filters for online trajectory planning with time- and frequency-domain specifications," Control Engineering Practice, vol. 20, no. 12, pp. 1385 - 1399, 2012.

[23] — , "Trajectory generation via fir filters: A procedure for timeoptimization under kinematic and frequency constraints," Control Engineering Practice, vol. 87, pp. 43 - 58, 2019.

[24] L. Biagiotti. (2020) Automatic design of an optimal trajectory generator in matlab/simulink. [Online]. Available: http://www.dii.unimore.it/ lbiagiotti/TrajectoryGeneratorNew.zip

[25] Y. Fang, J. Hu, W. Liu, Q. Shao, J. Qi, and Y. Peng, "Smooth and timeoptimal s-curve trajectory planning for automated robots and machines," Mechanism and Machine Theory, vol. 137, pp. 127 - 153, 2019.

[26] H. D. Tho, A. Kaneshige, and K. Terashima, "Minimum-time s-curve commands for vibration-free transportation of an overhead crane with actuator limits," Control Engineering Practice, vol. 98, p. 104390, 2020.

[27] K. Kozak, W. Singhose, and I. Ebert-Uphoff, "Performance measures for input shaping and command generation," Journal of Dynamic Systems Measurement and Control, vol. 128, no. 3, pp. 731-736, 2006.

[28] L. Biagiotti, C. Melchiorri, and L. Moriello, "Optimal trajectories for vibration reduction based on exponential filters," IEEE Transactions on Control Systems Technology, vol. 24, no. 2, pp. 609-622, 2016.

[29] Quanser, Rotary Flexible Link, 2020. [Online]. Available: https://www.quanser.com/products/rotary-flexible-link/

[30] W. He, X. He, M. Zou, and H. Li, "Pde model-based boundary control design for a flexible robotic manipulator with input backlash," IEEE Trans. on Control Systems Technology, vol. 27, no. 2, pp. 790-797, 2019.

[31] W. He, T. Wang, X. He, L. Yang, and O. Kaynak, "Dynamical modeling and boundary vibration control of a rigid-flexible wing system," IEEE/ASME Transactions on Mechatronics, pp. 1-1, 2020.

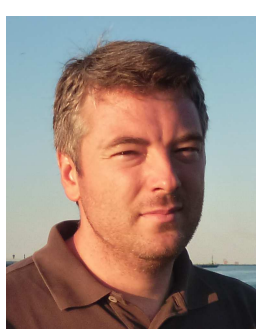

Luigi Biagiotti received the Laurea degree in Electronic Engineering from the University of Bologna in 2000 , and the $\mathrm{PhD}$ degree from the same University in 2003. Since September 2008, he joined the Department of Engineering "Enzo Ferrari" of the University of Modena and Reggio Emilia as Assistant Professor in the area of the Automatic Controls. His research interests include modelling and control of physical systems, trajectory planning and optimization, nonlinear control and control of robotic systems.

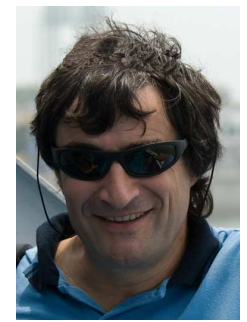

Claudio Melchiorri received the Laurea degree in Electronic Engineering at the University of Bologna, Italy, in 1985, and the PhD in 1990. Currently, he is Full Professor in Robotics at DEI, the Department of Electrical, Electronic and Information Engineering "Guglielmo Marconi" of the University of Bologna, Italy. He has served in the organization of several international conferences. He has been Editor in Chief of the IFAC Journal on Mechatronics in 20032008. He has published about 350 scientific papers and is author of two books on trajectory planning for automatic machines and robots, and author or (co-)editor of seven books in robotics. His research interests include dexterous manipulation, haptics, telemanipulation, nonlinear control, motion control. 